\title{
ON THE WEAK SOLUTIONS \\ OF THE FORWARD PROBLEM IN EEG
}

\author{
M. I. TROPAREVSKY AND D. RUBIO
}

Received 9 May 2003

The process underlying the generation of the EEG signals can be described as a set of current sources within the brain. The potential distribution produced by these sources can be measured on the scalp and inside the brain by means of an EEG recorder. There is a well-known mathematical model that relates the electric potential in the head with the intracerebral sources. In this paper, we study and prove some properties of the solutions of the model for known sources. In particular, we study the error in the potential, introduced by considering an approximated shape of the head.

\section{Introduction}

The electric process underlying the generation of the EEG can be described as a set of current sources within the brain. In the case of epilepsy, there are epileptogenic zones that give major contribution in the generation of the electric field and, for several decades, neurologists have been interested in solving the problem of determining the location and orientation of these current sources from the measured potential on the scalp. This problem is known as the inverse problem in EEG. A first step towards its solution is to solve the forward problem (FP) in EEG that consists in calculating the superficial potential for any possible configuration of the sources. A typical mathematical model that describes this process is a differential boundary value problem of second order, based on the static approximation of the Maxwell equations (see [5]). In order to calculate the solution, a simplified head model is adopted.

Copyright (c) 2003 Hindawi Publishing Corporation

Journal of Applied Mathematics 2003:12 (2003) 647-656

2000 Mathematics Subject Classification: 35Q60, 35D99, 35J05

URL: http:/ /dx.doi.org/10.1155/S1110757X03305030 
The human head is a complicated anisotropic media with tissues of different conductivity values. Usually it is modeled as three isotropic and homogeneous volumes representing brain, skull, and scalp. The choice of the head model is important in order to guarantee accurate solutions.

Some authors have calculated the solution in the case of spherical domains where it is possible to solve the differential boundary problem exactly by a series of functions (see $[1,2,8,9])$. Numerical solutions are usually tested against it taking into account only the errors introduced by the chosen numerical methods disregarding the fact that the domain has been approximated.

Up to our knowledge, the lack of accuracy introduced by considering the approximated domains instead of a real-shaped one has not been studied. In this paper, we explore some theoretical properties of the solution of the FP and establish theoretical bounds for the errors produced by the approximation mentioned above.

The paper is organized as follows: in Section 2 we present the differential system of equations that model the FP. The existence and uniqueness of solutions are stated in Section 3. Section 4 establishes a bound for the error that is introduced when solving the system on a domain that approximates the head. Finally, we present some conclusions.

\section{The mathematical model}

The electrical activity of the brain consists of currents generated by biochemical sources at the cellular level. The electric and magnetic fields that they produce can be estimated by means of Maxwell's equations (see $[5,6])$. Based on the properties of the tissues involved (see [6]), the velocity of propagation of the electromagnetic waves caused by potential changes within the brain is such that the effect of the potential changes may be detected simultaneously at any point in the brain or in the surrounding tissues. In consequence, the use of a static approximation of Maxwell's equations is justified. This approximation uncouples the equations for the magnetic and electric fields. Consequently, the second-order partial differential equation

$$
\nabla \cdot(\sigma(x) \nabla u(x))=\nabla \cdot J_{i}(x)
$$

relates the measured electric potential $u$ and the impressed current $J_{i}$, usually modeled as a dipole (associated with the microscopic currents). The function $\sigma(x)$ contains the value of the conductivity of the different tissues. 
Air is an insulating material that does not support current flow, therefore, the normal derivative to the head at the boundary must be zero:

$$
\frac{\partial u(x)}{\partial v}=0, \quad x \in \partial G
$$

where $G$ is the volume representing the head, $\partial G$ is its external surface, and $v$ represents the outward normal.

We assume that $G$ can be described as three homogeneous sets, each one surrounded by the next one, where the radii and conductivity values are given. We denote them from the inner one to the outer one: $G_{1}$ the brain, $G_{2}$ the skull, and $G_{3}$ the scalp. The surface between them are denoted by $S_{1}, S_{2}$, and $S_{3}$, respectively. Note that $S_{3}=\partial G$.

The function $\sigma(x)$ that contains the conductivity of the different tissues at each point is positive, usually assumed to be discontinuous and piecewise constant

$$
\sigma(x)= \begin{cases}\sigma_{1}, & x \in G_{1} \\ \sigma_{2}, & x \in G_{2} \\ \sigma_{3}, & x \in G_{3} \\ 0, & x \notin G .\end{cases}
$$

There are physical considerations that must be taken into account:

(i) the potential is continuous across the different regions;

(ii) the normal derivative of the potential is continuous across the different regions;

(iii) the scalp potential $u(x)$ is measured as a difference between the potential value at each point $x \in S_{3}$ and its value at a reference point $x_{0} \in S_{3}$.

If we denote by $[\cdot]$ the difference between the values of the functions inside the brackets through the indicated surface, they can be written as

$$
\begin{gathered}
{\left.[u]\right|_{S_{i}}=0,} \\
{\left.\left[\sigma(x) \frac{\partial u}{\partial n}\right]\right|_{S_{i}}=0,} \\
u\left(x_{0}\right)=0,
\end{gathered}
$$

respectively. 
Therefore, the resulting boundary value problem is

$$
\begin{gathered}
\nabla \cdot(\sigma(x) \nabla u(x))=\nabla \cdot J_{i}(x), \quad x \in G, \\
\frac{\partial u(x)}{\partial \mathcal{v}}=0, \quad x \in \partial G
\end{gathered}
$$

subject to

$$
\left.[u]\right|_{S_{i}}=0,\left.\quad\left[\sigma(x) \frac{\partial u}{\partial n}\right]\right|_{S_{i}}=0
$$

\section{Existence and uniqueness of solutions for the FP}

In order to assure the existence of solutions of (2.7) with boundary condition (2.8), we need to introduce some definitions and notations that will lead us to the definition of weak solution of (2.7) with boundary condition (2.8).

We denote by

$$
\langle f, g\rangle_{G}=\int_{G} f g d x
$$

the inner product in $G$.

Let $\|\cdot\|_{L^{n}(G)}$ be the norm in $L^{n}(G)$ :

$$
\|\phi\|_{L^{n}(G)}=\left(\int_{G}|\phi(x)|^{n} d x\right)^{1 / n}
$$

and $\|\cdot\|_{L^{\infty}(G)}$ the norm in $L^{\infty}(G)$ :

$$
\|\phi\|_{L^{\infty}(G)}=\operatorname{ess} \sup _{G}|\phi(x)| .
$$

We say that $f \in L^{n}(G)$ if $\|f\|_{L^{n}(G)}<\infty$, that is, if $f$ is essentially bounded.

We denote by $\mu(G)$ the Lebesgue measure of the set $G$.

Suppose that $u$ is a solution of (2.1) and multiply this equation by a function $v$. Assuming that both $u$ and $v$ are regular enough to apply the integral theorems to the resulting equation, the solution $u$ must verify the following identity:

$$
\langle\sigma \nabla u, \nabla v\rangle_{G}=-\left\langle\nabla \cdot J_{i}, v\right\rangle_{G^{\prime}}
$$

or, equivalently,

$$
\int_{G} \sigma(x) \nabla u \nabla v=-\int_{G} \nabla \cdot J_{i} v .
$$


A weak solution of (2.7) with boundary condition (2.8) is a function $u$ that verifies (3.5) for all functions $v$ with weak derivative of first order, that is, $v \in H^{1}$, where

$$
H^{1}(G)=\left\{v \in L^{2}(G) \mid \exists w \in L^{2}(G) \text { with } \int_{G} v D \phi d x=-\int_{G} \phi w d x\right\} .
$$

In this case, we say that $w$ is the first-order weak derivative of $v$.

Remark 3.1. Note that if $\sigma$ is piecewise constant and (2.5) is verified, then (3.5) is equivalent to

$$
\int_{G_{1}} \sigma_{1}(x) \nabla u \nabla v+\int_{G_{2}} \sigma_{2}(x) \nabla u \nabla v+\int_{G_{3}} \sigma_{3}(x) \nabla u \nabla v=-\int_{G} \nabla \cdot J_{i} v .
$$

From now on, we work with weak solutions since any classical solution to the problem is also a weak solution.

Proposition 3.2 (existence and uniqueness of weak solutions). There exists a unique solution to the boundary value problem (2.7), (2.8), and (2.9) that describes the FP in EEG, where $G=\cup_{i=1}^{3} G_{i}$, as described in Section 2, and $\sigma(x)$ is the piecewise constant function described in (2.3).

Proof. It can be proved (see $[3,7]$ ) that if $\sigma(x)$ is positive and piecewise $C^{1}$, there exist weak solutions, identical up to a constant, of the secondorder equation (2.7) subject to

$$
\int_{G} \nabla \cdot J_{i}=0
$$

or, equivalently,

$$
\int_{\partial G} J_{i}=0
$$

that is automatically fulfilled because $J_{i}$ has finite support inside $G_{1}$ (dipole). The uniqueness of solution of FP is justified since the potential verifies $u\left(x_{0}\right)=0$ at the reference point $x_{0}$ on the scalp.

Remark 3.3. The result of Proposition 3.2 remains valid if $G$ is composed by any number of sets $G_{i}$ and if $\sigma(x) \in C^{1}(G)$. 


\section{Solutions on different domains}

In this section, we consider that the domain where we solve (2.7) is composed by only one set. The conductivity function $\sigma(x)$ need not be constant in the domain, actually $\sigma(x) \in C^{1}$ and is positive if required.

Let $G$ and $H$ be two sets representing the head (see Figure 4.1).

We consider that

(i) $u_{G}$ is a weak solution of (2.1) on $G$ :

$$
\nabla\left(\sigma_{G}(x) \nabla u_{G}(x)\right)=\nabla \cdot J_{i}(x)
$$

(ii) $u_{H}$ is a weak solution of (2.1) on $H$ :

$$
\nabla \cdot\left(\sigma_{H}(x) \nabla u_{H}(x)\right)=\nabla \cdot J_{i}(x)
$$

where $\sigma_{G}(x)$ and $\sigma_{H}(x)$ are the conductivity functions in $G$ and $H$, and supp $J_{i} \subset G \cap H$. We prove that if the difference between the sets $G$ and $H$ is small, so it is the $L^{2}$-norm of the difference between the solutions $u_{G}$ and $u_{H}$. To do so, we calculate a bound for the $L^{2}$-norm of the difference of the solutions for the two different domains $G$ and $H$. We consider that the conductivity functions $\sigma_{G}$ and $\sigma_{H}$ are positive, coincide on $G \cap H$, and verify

$$
\sigma_{G}(x)=\left\{\begin{array}{ll}
\sigma(x), & x \in G, \\
0, & x \notin G,
\end{array} \quad \sigma_{H}(x)= \begin{cases}\sigma(x), & x \in H \\
0, & x \notin H .\end{cases}\right.
$$

We denote by $G \Delta H$ the symmetric difference between the domains $G$ and $H$, that is, $G \triangle H=(G-H) \cup(H-G)$. We consider that $G, H$ are bounded subsets of $R^{3}, \partial G \in C^{1}, \partial H \in C^{1}$.

We assume that $\nabla u_{G}$ and $\nabla u_{H}$ are bounded in $G$ and $H$, respectively. In the case of the solutions of the FP in EEG, this assumption is reasonable since $u_{G}$ and $u_{H}$ represent the electric potential on the head, and consequently, $\nabla u_{G}$ and $\nabla u_{H}$ are the electric fields.

In order to establish a bound for $\left\|u_{G}-u_{H}\right\|_{L^{2}(G \cap H)}$, we need some lemmas.

LEMMA 4.1. Let $u_{G}$ and $u_{H}$ be solutions of (4.1) and (4.2), respectively. Then, for every open and bounded subset of $V \subset R^{3}, G \cup H \subset V$, there exists an extension $u$ of $u_{G}-u_{H}$ to $R^{3}$ such that

(1) $\|u\|_{L^{2}(G \cup H)} \leq C\|\nabla u\|_{L^{2}(V)}$ for a constant $C$ that does not depend on $u$;

(2) if, in addition, $\left\|\nabla u_{G}\right\|_{L^{\infty}(G)}$ and $\left\|\nabla u_{H}\right\|_{L^{\infty}(H)}$ are finite, then $u \in$ $L^{\infty}(V)$. 


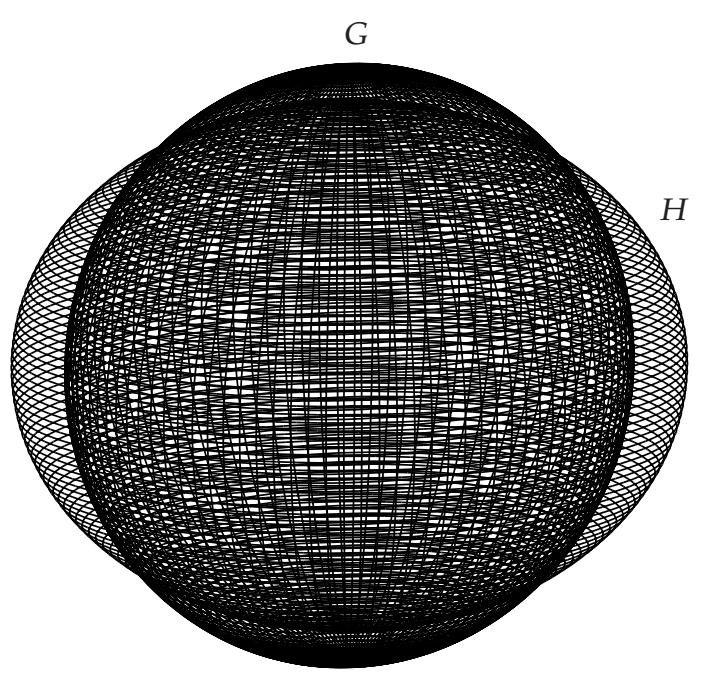

FigURE 4.1

Proof. Let $G \cup H \subset V, \bar{u}_{G}$, and $\bar{u}_{H}$ be the extensions of $u_{G}$ and $u_{H}$, respectively, to $R^{3}$ that verify (see [4])

$$
\begin{array}{cc}
\left.\bar{u}_{G}\right|_{G}=\left.u_{G}\right|_{G^{\prime}} \quad & \left.\bar{u}_{G}\right|_{R^{3}-V}=0, \\
\left.\bar{u}_{H}\right|_{H}=\left.u_{H}\right|_{H^{\prime}} & \left.\bar{u}_{H}\right|_{R^{3}-V}=0 .
\end{array}
$$

If we define $u=\bar{u}_{G}-\bar{u}_{H}$, it has compact support and $\left.u\right|_{R^{3}-V}=0$. From the Poincaré inequality (see [4]), we have

$$
\|u\|_{L^{2}(V)} \leq C\|\nabla u\|_{L^{2}(V)} .
$$

In addition, $\|u\|_{L^{2}(G \cup H)} \leq\|u\|_{L^{2}(V)}$. Combining these inequalities, the first statement of the lemma follows.

Since we assume that $\left\|\nabla u_{G}\right\|_{L^{\infty}(G)}$ and $\left\|\nabla u_{H}\right\|_{L^{\infty}(H)}$ are finite, then $\nabla \bar{u}_{G}, \nabla \bar{u}_{H} \in L^{\infty}(V)$, and $\nabla u \in L^{\infty}(V)$, hence the lemma follows.

Lemma 4.2. Under the hypothesis of Lemma 4.1, we can choose $V$ such that

$$
\|\nabla u\|_{L^{2}(V)} \leq K \mu(G \Delta H)^{1 / 2}
$$

for some constant $K$. 
654 On the weak solutions of the forward problem in EEG

Proof. Choose $V$ such that $G \cup H \subset V$ and $\mu(V-(G \cup H)) \leq \mu(G \triangle H)$, then

$$
\|\nabla u\|_{L^{2}(V)}^{2}=\|\nabla u\|_{L^{2}(V-(G \cup H))}^{2}+\|\nabla u\|_{L^{2}(G \Delta H)}^{2}+\|\nabla u\|_{L^{2}(G \cap H)}^{2} .
$$

Since $\operatorname{supp}\left(\nabla \cdot J_{i}\right) \subset(G \cup H)$ and $u_{G}, u_{H}$ are weak solutions of (2.7) in $G$ and $H$, respectively, from (3.5) we obtain

$$
\int_{G} \sigma_{G}(x) \nabla u_{G} \nabla v=\int_{H} \sigma_{H}(x) \nabla u_{H} \nabla v, \quad \forall v \in H^{1}(V) .
$$

Let $\sigma(x)=\sigma_{G}(x)-\sigma_{H}(x)$. Choosing $v=u$ yields

$$
\int_{G \cap H} \sigma(x) \nabla\left(u_{G}-u_{H}\right) \nabla u=\int_{H-G} \sigma_{H}(x) \nabla u_{H} \nabla u-\int_{G-H} \sigma_{G}(x) \nabla u_{G} \nabla u .
$$

Let $\sigma_{M}=\max \{\sigma(x), x \in G \cup H\}$ and $\sigma_{m}=\min \{\sigma(x), x \in G \cup H\}$ and let $S_{G}, S_{H}$, and $S$ be bounds for $\left\|\nabla u_{G}\right\|,\left\|\nabla u_{H}\right\|$, and $\|\nabla u\|$, respectively. From (4.9), it follows that

$$
\sigma_{m}\left\|\nabla\left(u_{G}-u_{H}\right)\right\|_{L^{2}(G \cap H)}^{2} \leq \sigma_{M} S_{H} S \mu(H-G)+\sigma_{M} S_{G} S \mu(G-H),
$$

and consequently, there exists a constant $C$ such that

$$
\left\|\nabla\left(u_{G}-u_{H}\right)\right\|_{L^{2}(G \cap H)}^{2} \leq C \mu(G \Delta H) .
$$

Finally, combining equations (4.7) and (4.11), we obtain

$$
\|\nabla u\|_{L^{2}(V)}^{2} \leq S^{2}(\mu(V-(G \cup H))+\mu(G \Delta H))+C \mu(G \Delta H) .
$$

Now, for the chosen $V$, the thesis follows.

The following result is a consequence of Lemmas 4.1 and 4.2.

LEMMA 4.3. For $u$ and $V$ defined above, there is a constant $M$ not depending on $u$ such that

$$
\|u\|_{L^{2}(G \cup H)} \leq M \mu(G \triangle H)^{1 / 2} .
$$

Proof. From Lemma 4.1, $\|u\|_{L^{2}(G \cup H)} \leq C\|\nabla u\|_{L^{2}(V)}$ and from Lemma 4.2, $\|\nabla u\|_{L^{2}(V)} \leq K \mu(G \triangle H)^{1 / 2}$. Setting $M=C K,(4.13)$ is verified. 
Now we apply the result of Lemma 4.3 to the case of the EEG signals. The following proposition shows that the difference of two solutions of the FP calculated on different domains approaches zero when the measure of the symmetric difference of their domains tends to zero.

Proposition 4.4. Let $G$ and $H$ be two different domains in $R^{3}$ that models the head. Let $u_{G}$ and $u_{H}$ be the solutions of the FP in EEG described by (4.1) and (4.2), respectively, and $\bar{u}_{G}$ and $\bar{u}_{H}$ the extensions of these solutions to $R^{3}$ presented in Lemma 4.1. Then

$$
\left\|\bar{u}_{G}-\bar{u}_{H}\right\|_{L^{2}(G \cup H)}^{2} \longrightarrow 0 \quad \text { when } \mu(G \triangle H) \longrightarrow 0 .
$$

Proof. The solutions $u_{G}$ and $u_{H}$ represent the electric potentials in the domains $G$ and $H$, respectively, thus $\nabla u_{G}=E_{G}$ and $\nabla u_{H}=E_{H}$ are the electric fields on $G$ and $H$ that are bounded on $R^{3}$. Since all the hypothesis of the previous lemmas hold, there exist a constant $M$ such that

$$
\left\|u_{G}-u_{H}\right\|_{L^{2}(G \cup H)}^{2} \leq M \mu(G \triangle H)^{1 / 2} .
$$

Consequently, $\left\|u_{G}-u_{H}\right\|_{L^{2}(G \cup H)}^{2} \rightarrow 0$ when $\mu(G \Delta H) \rightarrow 0$.

Inequality (4.15) means that the error produced by considering weak solutions of (2.7) in two different domains, with conductivity function verifying (4.3), is proportional to the Lebesgue measure of the symmetric difference of those domains.

Remark 4.5. The result of Proposition 4.4 can be extended to the case where $G$ is a multicompartment set and $\sigma(x)$ is a $C^{1}$ piecewise positive function that verifies (2.5).

\section{Conclusions}

In this paper, we state and prove some theoretical properties of the weak solution of the equations that model the FP in EEG.

Proposition 4.4 states that if (2.7) is solved in two different domains $G$ and $H$, the $L^{2}$-norm of the difference between the solutions tends to zero when $\mu(G \triangle H)$ tends to zero. This result is concerned with a real situation: the dimensions and shape of the head are approximated. It is important from a theoretical and qualitative point of view since it gives us some confidence on the solutions obtained in the case of approximated shape and dimensions of the head. 
656 On the weak solutions of the forward problem in EEG

\section{References}

[1] J. C. de Munck, The potential distribution in a layered anisotropic spheroidal volume conductor, J. Appl. Phys. 64 (1988), 464-470.

[2] J. C. de Munck and M. J. Peters, A fast method to compute the potential in the multisphere model, IEEE Trans. Biomed. Eng. 40 (1993), no. 11, 1166-1174.

[3] A. El Badia and T. Ha-Duong, An inverse source problem in potential analysis, Inverse Problems 16 (2000), no. 3, 651-663.

[4] L. C. Evans, Partial differential equations, Graduate Studies in Mathematics, vol. 19, American Mathematical Society, Rhode Island, 1998.

[5] M. Hamalainen, R. Hari, R. J. Ilmoniemi, J. Knuutila, and O. Lounasmaa, Magnetoencephalography, theory, instrumentation and applications to noninvasive studies of the working human brain, Rev. Modern Phys. 65 (1993), 413487.

[6] F. Lopes da Silva and A. Van Rotterdam, Biophysical aspects of EEG and magnetoencephalogram generation, Electroencephalography: Basic Principles, Clinical Applications, and Related Fields, Williams and Wilkins, Maryland, 1999, pp. 93-109.

[7] V. P. Mijailov, Ecuaciones Diferenciales en Derivadas Parciales [Partial Differential Equations], Editorial Mir, Moscow, 1978 (Spanish), translated from the Russian by K. P. Medkov.

[8] J. C. Mosher, R. M. Leahy, and P. S. Lewis, EEG and MEG: forward solutions for inverse methods, IEEE Trans. Biomed. Eng. 46 (1999), no. 3, 245-259.

[9] Z. Zhang, A fast method to compute surface potentials generated by dipoles within multilayer anisotropic spheres, Phys. Med. Biol. 40 (1995), 335-349.

M. I. Troparevsky: Departamento de Matemática, Facultad de Ingeniería, Universidad de Buenos Aires, Paseo Colon 850, Buenos Aires, Argentina

E-mail address: mitropa@fi.uba.ar

D. Rubio: Escuela de Ciencia y Tecnología, Universidad Nacional de General San Martín, San Lorenzo 3391, Pcia. Buenos Aires, Argentina

Current address: Instituto de Ciencias, Universidad Nacional de General Sarmiento, J.M. Gutierrez 1613, Pcia. Buenos Aires, Argentina

E-mail address: arubio@ungs.edu.ar 


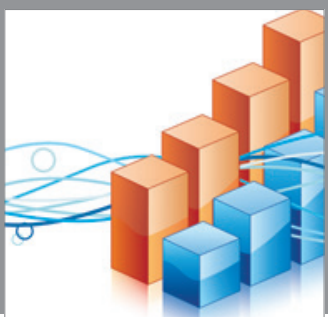

Advances in

Operations Research

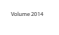

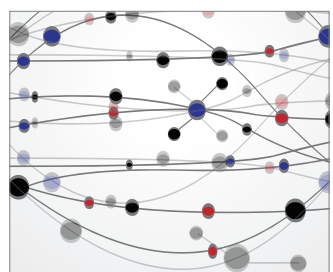

\section{The Scientific} World Journal
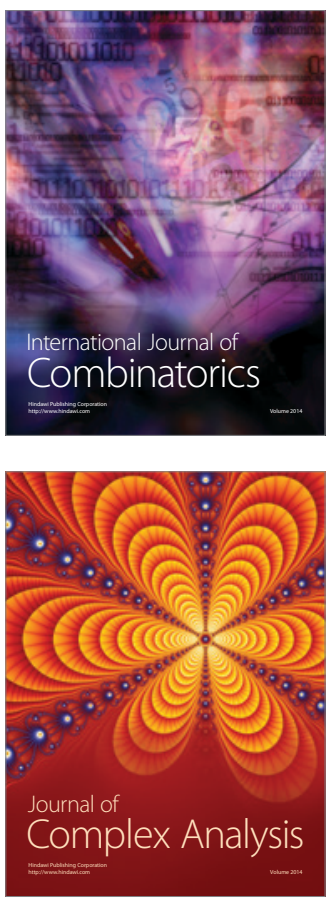

International Journal of

Mathematics and

Mathematical

Sciences
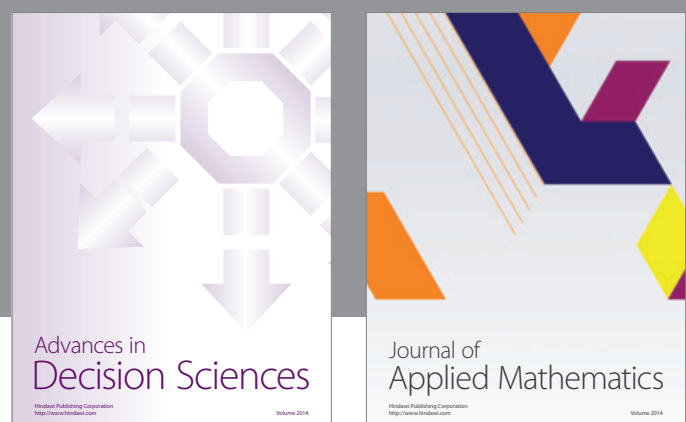

Journal of

Applied Mathematics
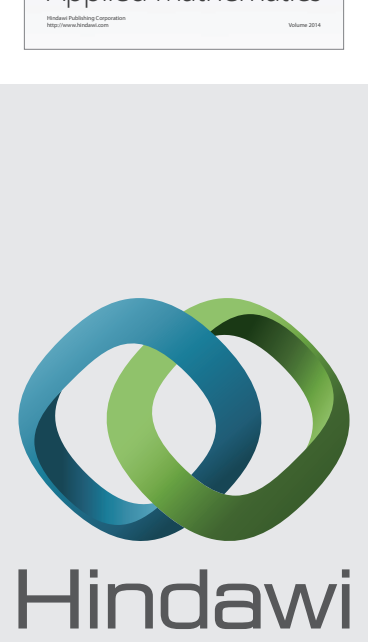

Submit your manuscripts at http://www.hindawi.com
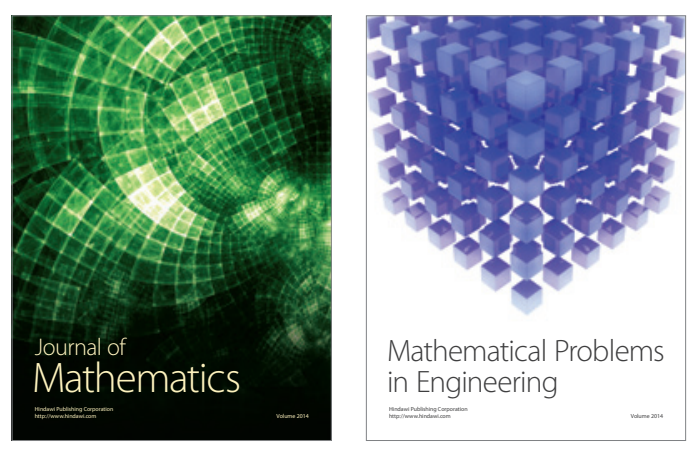

Mathematical Problems in Engineering
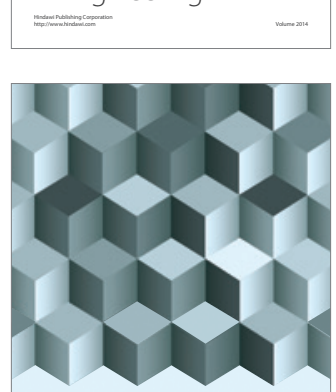

Journal of

Function Spaces
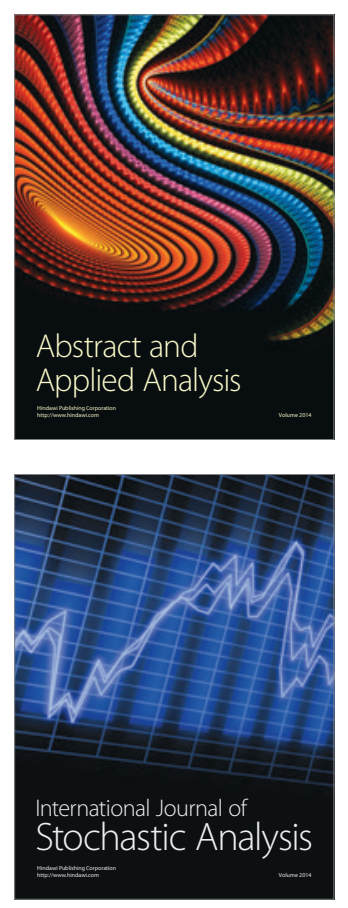

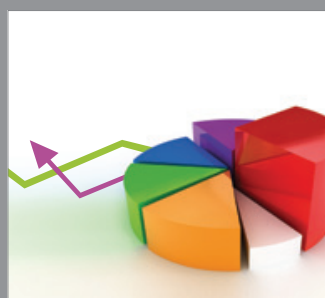

ournal of

Probability and Statistics

Promensencen
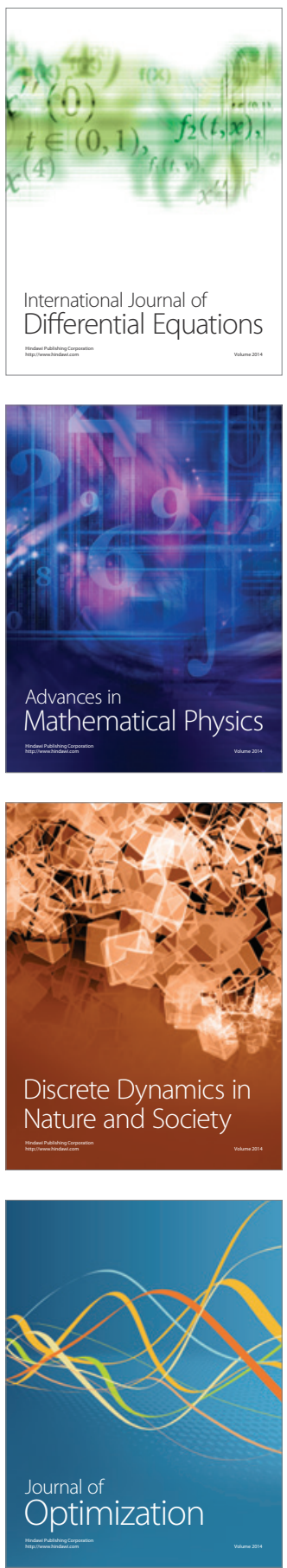